\title{
openheart Calibrated integrated backscatter and myocardial fibrosis in patients undergoing cardiac surgery
}

David L Prior, ${ }^{1,2,3}$ Jithendra B Somaratne, ${ }^{1}$ Alicia J Jenkins, ${ }^{2}$ Michael Yii, ${ }^{4,5}$ Andrew E Newcomb, ${ }^{4,5}$ Casper G Schalkwijk, ${ }^{6}$ Mary J Black, ${ }^{7}$ Darren J Kelly, ${ }^{2}$ Duncan J Campbell ${ }^{2,3}$

To cite: Prior DL, Somaratne JB, Jenkins AJ, et al. Calibrated integrated backscatter and myocardial fibrosis in patients undergoing cardiac surgery. Open Heart 2015;2:e000278. doi:10.1136/openhrt-2015000278

- Additional material is available. To view please visit the journal (http://dx.doi.org/ 10.1136/openhrt-2015000278)

Received 8 April 2015 Revised 16 June 2015 Accepted 31 July 2015
CrossMark

For numbered affiliations see end of article.

Correspondence to Professor David L Prior; David.PRIOR@svhm.org.au

\section{ABSTRACT}

Objective: The reported association between calibrated integrated backscatter (clB) and myocardial fibrosis is based on study of patients with dilated or hypertrophic cardiomyopathy and extensive (mean 15-34\%) fibrosis. Its association with lesser degrees of fibrosis is unknown. We examined the relationship between $\mathrm{ClB}$ and myocardial fibrosis in patients with coronary artery disease.

Methods: Myocardial histology was examined in left ventricular epicardial biopsies from 40 patients (29 men and 11 women) undergoing coronary artery bypass graft surgery, who had preoperative echocardiography with $\mathrm{ClB}$ measurement.

Results: Total fibrosis (picrosirius red staining) varied from $0.7 \%$ to $4 \%$, and in contrast to previous reports, CIB showed weak inverse associations with total fibrosis $(r=-0.32, p=0.047)$ and interstitial fibrosis $(r=-0.34, p=0.03)$. However, clB was not significantly associated with other histological parameters, including immunostaining for collagens I and III, the advanced glycation end product (AGE) $\mathrm{N}^{\varepsilon}$-(carboxymethyl)lysine (CML) and the receptor for AGEs (RAGE). When biomarkers were examined, CIB was weakly associated with log plasma levels of amino-terminal pro-B-type natriuretic peptide $(r=0.34$, $p=0.03)$, creatinine $(r=0.33, p=0.04)$ and glomerular filtration rate $(r=-0.33, p=0.04)$, and was more strongly associated with log plasma levels of soluble vascular endothelial growth factor receptor-1 (sVEGFR1) $(r=0.44, p=0.01)$ and soluble RAGE $(r=0.53$, $\mathrm{p}=0.002$ ).

Conclusions: Higher clB was not a marker of increased myocardial fibrosis in patients with coronary artery disease, but was associated with higher plasma levels of SVEGFR-1 and soluble RAGE. The role of ClB as a non-invasive index of fibrosis in clinical studies of patients without extensive fibrosis is, therefore, questionable.

\section{INTRODUCTION}

Researchers and clinicians need a method to identify and quantify the extent of myocardial fibrosis throughout all stages of myocardial disease, so that they can study

\section{KEY QUESTIONS}

What is already known about this subject?

- Increased calibrated integrated backscatter (CIB) is associated with increased myocardial fibrosis in patients with dilated or hypertrophic cardiomyopathy and extensive (mean 15-34\%) fibrosis. However, its association with lesser degrees of fibrosis is unknown.

What does this study add?

- This is the first study to examine the relationship between $\mathrm{ClB}$ and myocardial fibrosis in patients with lesser degrees of fibrosis $(0.7-4 \%)$. A key novel finding was that increased $\mathrm{ClB}$ was not associated with increased myocardial fibrosis, but was associated with higher plasma levels of soluble vascular endothelial growth factor receptor-1 and soluble receptor for advanced glycation end products.

How might this impact on clinical practice?

- These data question whether $\mathrm{ClB}$ is a reliable non-invasive index of fibrosis in clinical studies of patients without extensive fibrosis.

longitudinal changes in myocardial fibrosis and measure the effects of novel antifibrotic therapies, particularly in the early stages of the disease when changes may be more likely to be reversible. One non-invasive approach to measurement of myocardial fibrosis has been the echocardiographic measurement of calibrated integrated backscatter (cIB) ${ }^{1-7}$

cIB is a measure of the ultrasonic reflectivity of the myocardium relative to the high reflectivity of the pericardium and the low reflectivity of blood. cIB is correlated with histologically measured myocardial fibrosis in patients with dilated or hypertrophic cardiomyopathy and extensive fibrosis (mean 15$34 \%$ fibrosis $),{ }^{1-5}$ and it has been applied to the non-invasive assessment of myocardial fibrosis in a broad spectrum of patients, including those with the metabolic syndrome 
and type 2 diabetes. ${ }^{6} 7$ Moreover, some authors have interpreted the association of cIB with left ventricular functional abnormalities as evidence for the contribution of myocardial fibrosis to these abnormalities. ${ }^{6} 7$ There is, however, uncertainty about the relationship between cIB and fibrosis in patients with less extensive fibrosis. We have shown that left ventricular functional abnormalities of the metabolic syndrome and type 2 diabetes are not associated with increased myocardial fibrosis. ${ }^{8}$ In addition, factors other than fibrosis may influence cIB. ${ }^{9-16}$ cIB increases in response to acute myocardial ischaemia ${ }^{9}$ and mild or greater degrees of acute cardiac allograft rejection, ${ }^{10}$ and is associated with serum levels of the angiogenesis-related biomarker interleukin-13 (IL-13). ${ }^{11}$ In patients with chronic renal failure, cIB is correlated with serum creatinine ${ }^{12}$ and is reduced by increased hours/week of dialysis over 12 months, ${ }^{13}$ but not by a single dialysis session. ${ }^{14}$ In addition, cIB is increased in overweight individuals ${ }^{15}$ and reduced by weight loss. ${ }^{16}$ Of note is the lack of correlation between CIB and T1 mapping techniques of cardiac MRI that have been proposed as an alternative measure of myocardial fibrosis. ${ }^{17}$

Given the uncertain relationship between cIB and myocardial fibrosis in patients without extensive fibrosis, we examined the relationship between cIB, myocardial fibrosis, clinical parameters and plasma levels of angiogenesis-related biomarkers in patients undergoing coronary artery bypass graft (CABG) surgery, who did not have dilated or hypertrophic cardiomyopathy.

\section{METHODS}

\section{Patient population and data collection}

The population consisted of all patients undergoing CABG surgery recruited to the St. Vincent's Hospital Melbourne Cardiac Tissue Bank who did not have heart failure or atrial fibrillation, had a relatively preserved left ventricular systolic function, and had a detailed echocardiographic examination, including cIB measurement, a few days before surgery. Details of the Tissue Bank are described elsewhere. ${ }^{18}$ From each patient recruited to the Tissue Bank, an epicardial partial-thickness wedgeshaped biopsy was taken immediately after cardioplegia from a region in the lateral wall of the left ventricle near the base of the heart, between the territories of the left anterior descending and circumflex arteries that was free of any macroscopic pathology, and without evidence of ischaemia or wall motion abnormality on preoperative or intraoperative imaging studies. None of the patients had dilated or hypertrophic cardiomyopathy. This study was approved by St. Vincent's Hospital Melbourne Human Research Ethics Committee and conforms to the principals outlined in the Declaration of Helsinki. Each participant provided written informed consent.

Details of haemodynamic measurements, and biochemical and histological methods are described in the online supplementary data.

\section{Echocardiographic evaluation}

All echocardiographic analyses were performed by the same individual who was blinded to myocardial histology, haemodynamic data and angiogenesis-related biomarker levels.

Transthoracic echocardiograms were performed with patients in the left lateral position using a VIVID 7 echocardiograph (GE Medical, Milwaukee, Wisconsin, USA). Routine M-mode, two-dimensional and Doppler images were acquired, and all images were stored in digital raw data format for subsequent offline analysis using commercially available software (EchoPAC PC, GE, Horten, Norway). All patients were in sinus rhythm and three cardiac cycles were captured for each measurement. Two-dimensional images of the left ventricle were acquired at sufficient frame rate to allow measurement of myocardial strain by speckle tracking from the fourchamber view. Chamber sizes and systolic function were measured according to the American Society of Echocardiography (ASE) guidelines. ${ }^{19}$ Left ventricular ejection fraction was measured using a biplane Simpson's method. Mitral inflow and pulmonary venous flow velocities were measured using pulsed wave Doppler with the transducer positioned at the apex according to the ASE guidelines. ${ }^{20}$ Mitral annular velocities were measured with the transducer at the apex and with placement of the sample volume over the septal and lateral mitral annulus. Peak systolic $\left(\mathrm{S}^{\prime}\right)$, early diastolic $\left(\mathrm{E}^{\prime}\right)$ and late diastolic $\left(\mathrm{A}^{\prime}\right)$ velocities were measured at the septal and lateral annulus, and averaged to provide mean annular velocities. Strain and strain rate were measured in the six-wall segments seen in the apical four-chamber view using speckle tracking within the EchoPAC software. The peak global strain and strain rate are the peak values of the instantaneous mean of all six segments. The mean peak systolic strain and strain rate are the mean of the peak values in all segments and were, therefore, not acquired at the same time due to temporal variation between segments.

cIB was measured from a parasternal long axis view with care taken to ensure the wall was perpendicular to the transducer. Myocardial signal intensity was measured at end diastole using a $4 \times 8 \mathrm{~mm}$ region of interest within the mid-wall of the basal anteroseptum and the basal inferolateral wall, and from the posterior pericardium. The cIB value was calculated by subtracting the pericardial intensity from the average of the anteroseptal and basal inferolateral wall intensities, and represents the mean of two separate calculations performed 24 months apart by the same investigator with an intraclass correlation of $0.85(95 \%$ CI 0.73 to $0.92, \quad \mathrm{p}<0.001)$. A Bland-Altman plot for the two measurements of cIB is shown in the online supplementary data.

\section{Statistical analysis}

Data are presented as means \pm SD for normally distributed variables and as medians with 25 th and 75 th centiles for variables that were not normally distributed. The 
Table 1 Clinical and biochemical characteristics, and their correlation with calibrated integrated backscatter in 29 men and 11 women with coronary artery disease

\begin{tabular}{|c|c|c|c|}
\hline Variable & $\begin{array}{l}\text { Mean } \pm S D \text { or median } \\
(\text { IQR })(n=40)\end{array}$ & $\mathbf{r}$ & p Value \\
\hline Age (years) & $65 \pm 11$ & 0.31 & 0.053 \\
\hline Weight (kg) & $83 \pm 13$ & 0.003 & 0.99 \\
\hline Height $(\mathrm{cm})$ & $168 \pm 10$ & -0.05 & 0.75 \\
\hline Body surface area $\left(\mathrm{m}^{2}\right)$ & $1.9 \pm 0.2$ & -0.01 & 0.94 \\
\hline Body mass index $\left(\mathrm{kg} / \mathrm{m}^{2}\right)$ & $30 \pm 6$ & 0.02 & 0.90 \\
\hline Systolic blood pressure (mm Hg) & $134 \pm 15$ & 0.06 & 0.72 \\
\hline Diastolic blood pressure $(\mathrm{mm} \mathrm{Hg})$ & $76 \pm 8$ & 0.04 & 0.83 \\
\hline Pulse pressure $(\mathrm{mm} \mathrm{Hg})$ & $58 \pm 14$ & 0.04 & 0.80 \\
\hline Fasting plasma glucose (mmol/L) & $6.0(5.4-6.5)^{*}$ & $-0.10 \dagger$ & $0.53 \dagger$ \\
\hline Fasting plasma insulin (pmol/L) & $65(36-108)^{\star}$ & $0.02 \dagger$ & $0.93 \dagger$ \\
\hline$\beta$-cell function from HOMA2-\%B & $64(52-110)^{*}$ & $0.08 \dagger$ & $0.61 \dagger$ \\
\hline Insulin sensitivity from HOMA2-\%S & $84(51-139)^{*}$ & $-0.008 \dagger$ & $0.96 \dagger$ \\
\hline Insulin resistance from HOMA2-IR & $1.2(0.8-2.0)^{\star}$ & $0.004 \dagger$ & $0.98 \dagger$ \\
\hline Plasma NT-proBNP (pmol/L) & $16(7-32)^{*}$ & $0.34 \dagger$ & $0.03 \dagger$ \\
\hline Plasma creatinine $(\mu \mathrm{mol} / \mathrm{L})$ & $87(77-110)^{\star}$ & $0.33 \dagger$ & $0.04 \dagger$ \\
\hline eGFR $\left(\mathrm{mL} / \mathrm{min} / 1.73 \mathrm{~m}^{2}\right)$ & $68 \pm 16$ & -0.33 & 0.04 \\
\hline Plasma $\mathrm{C}$ reactive protein (mg/L) & $2.8(0.7-7.2)^{\star}$ & $0.03 \uparrow$ & $0.87 \dagger$ \\
\hline Plasma PIP $(\mu \mathrm{g} / \mathrm{L})$ & $284 \pm 78$ & 0.07 & 0.68 \\
\hline Plasma PINP $(\mu \mathrm{g} / \mathrm{L})$ & $32 \pm 14$ & 0.01 & 0.95 \\
\hline Plasma PIIINP $(\mu \mathrm{g} / \mathrm{L})$ & $2.5 \pm 1.1$ & 0.03 & 0.84 \\
\hline \multicolumn{4}{|c|}{$\begin{array}{l}\text { eGFR is calculated from the Modification of Diet in Renal Disease formula. }{ }^{29} \\
\text { HOMA2-\%B; HOMA2-\%S and HOMA2-IR were calculated using the HOMA calculator V.2.2. }{ }^{30} \\
\text { `Median value and IQR given non-parametric distribution. } \\
\text { tValues derived from log-transformed data. } \\
\text { eGFR, estimated glomerular filtration rate; HOMA2-\%B, } \beta \text {-cell function; HOMA2-IR, insulin resistance; HOMA2-\%S, insulin sensitivity; } \\
\text { NT-proBNP, amino-terminal-pro-B-type natriuretic peptide; PIIINP, intact amino-terminal propeptide of type III procollagen; PINP, intact } \\
\text { amino-terminal propeptide of type } 1 \text { procollagen; PIP, carboxy-terminal propeptide of procollagen type I. }\end{array}$} \\
\hline
\end{tabular}

normality of continuous data was verified with the Kolmogorov-Smirnov test and variables with a positively skewed distribution were log transformed before analysis. Correlations were estimated by univariable linear least squares analysis, and Pearson or Spearman correlation coefficients were estimated. Differences were considered significant at two-tailed $\mathrm{p}<0.05$.

\section{RESULTS}

\section{Clinical characteristics}

The clinical and biochemical characteristics of the patient population are shown in table 1 . Of the 40 patients (29 men and 11 women), 25 had no evidence of previous myocardial infarction (no history of myocardial infarction or pathological $Q$ waves on ECG, akinesis or dyskinesis on ventriculogram or echocardiography, or scars visible on inspection of the heart at surgery); 9 had recently documented non-ST-segment-elevation myocardial infarction 5-89 days before surgery, and 6 had previous ST-segment elevation myocardial infarction before surgery. Eleven patients $(27.5 \%)$ were diabetic (1 type 1 and 10 type 2$), 17(42.5 \%)$ without diabetes had metabolic syndrome, and $12(30 \%)$ had neither diabetes nor metabolic syndrome according to the International Diabetes Federation criteria. All had extensive coronary artery disease and received a median of 3 (range 2-5) bypass grafts. Most were receiving recommended cardiovascular therapies. Of the 40 patients, $39(98 \%)$ were receiving aspirin, $38(95 \%)$ were receiving statins, $32(80 \%)$ were receiving $\beta$-blockers, $29(73 \%)$ were receiving ACE inhibitors and/or angiotensin receptor blockers, $11(28 \%)$ were receiving calcium antagonists, 9 $(23 \%)$ were receiving thiazides or indapamide, $9(23 \%)$ were receiving long-acting nitrates, and 2 (5\%) were receiving oral furosemide. None were receiving spironolactone or eplerenone therapy.

\section{Cardiac morphology and function}

All patients were in sinus rhythm with an ejection fraction $>52 \%$ (table 2). Mean interventricular septal thickness was $11 \pm 2 \mathrm{~mm}$, posterior wall thickness was $10 \pm 2 \mathrm{~mm}$ and left ventricular diastolic diameter was $47 \pm 6 \mathrm{~mm}$. Right ventricular function was normal in all participants. Left atrial area was $23.3 \pm 4.4 \mathrm{~cm}^{2}$, with 32 participants having left atrial enlargement (area $>20 \mathrm{~cm}^{2}$ ). Mean right atrial area was $17.4 \pm 3.9 \mathrm{~cm}^{2}$. Of the 39 participants in whom diastolic function could be assessed, 22 had a normal pattern, 12 had impaired relaxation and 5 had pseudonormal filling.

Mean cIB for the 40 patients was $-19.5 \pm 4.2 \mathrm{~dB}$ and varied from -29 to $-10 \mathrm{db}$. Mean cIB did not differ between men $(-19.4 \pm 3.9)$ and women $(-19.8 \pm 5.1)$. There were statistically significant correlations between cIB and log plasma amino-terminal-pro-B-type natriuretic 
Table 2 Echocardiographic and haemodynamic characteristics, and their correlation with calibrated integrated backscatter in 29 men and 11 women with coronary artery disease

\begin{tabular}{|c|c|c|c|}
\hline Variable & $\begin{array}{l}\text { Mean } \pm \text { SD or median } \\
\text { (IQR) }(n=30-40)\end{array}$ & $r$ & p Value \\
\hline Left ventricular ejection fraction (\%) & $65 \pm 7$ & 0.02 & 0.91 \\
\hline E/A ratio & $1.06 \pm 0.35$ & -0.03 & 0.87 \\
\hline Mean $\mathrm{S}^{\prime}(\mathrm{cm} / \mathrm{s})$ & $7.4 \pm 1.5$ & 0.03 & 0.87 \\
\hline Mean $\mathrm{E}^{\prime}(\mathrm{cm} / \mathrm{s})$ & $7.0 \pm 1.7$ & -0.06 & 0.70 \\
\hline Mean $A^{\prime}(\mathrm{cm} / \mathrm{s})$ & $9.0 \pm 1.9$ & -0.32 & 0.048 \\
\hline Average $E / E^{\prime}$ ratio & $11.2 \pm 3.5$ & 0.20 & 0.22 \\
\hline Peak global systolic strain (\%) & $-18.6 \pm 2.8$ & 0.14 & 0.45 \\
\hline Peak global systolic strain rate $\left(\mathrm{s}^{-1}\right)$ & $-0.97 \pm 0.17$ & 0.13 & 0.49 \\
\hline Mean peak systolic strain (\%) & $-19.2 \pm 2.8$ & 0.10 & 0.60 \\
\hline Mean peak systolic strain rate $\left(\mathrm{s}^{-1}\right)$ & $-1.21 \pm 0.44$ & -0.14 & 0.47 \\
\hline Calculated integrated backscatter (dB) & $-19.5 \pm 4.2$ & & \\
\hline Central venous pressure $(\mathrm{mm} \mathrm{Hg})$ & $7.8 \pm 3.4$ & -0.21 & 0.20 \\
\hline Pulmonary capillary wedge pressure $(\mathrm{mm} \mathrm{Hg})$ & $10(8-12)^{*}$ & $-0.24 \dagger$ & $0.13 \dagger$ \\
\hline Cardiac index $\left(\mathrm{L} / \mathrm{min} / \mathrm{m}^{2}\right)$ & $2.6 \pm 0.6$ & 0.17 & 0.30 \\
\hline
\end{tabular}

peptide (NT-proBNP) level, plasma creatinine, estimated glomerular filtration rate (eGFR) (table 1), and mean peak late diastolic annular velocity (A') (table 2). However, cIB was not significantly correlated with plasma levels of carboxy-terminal propeptide of procollagen type
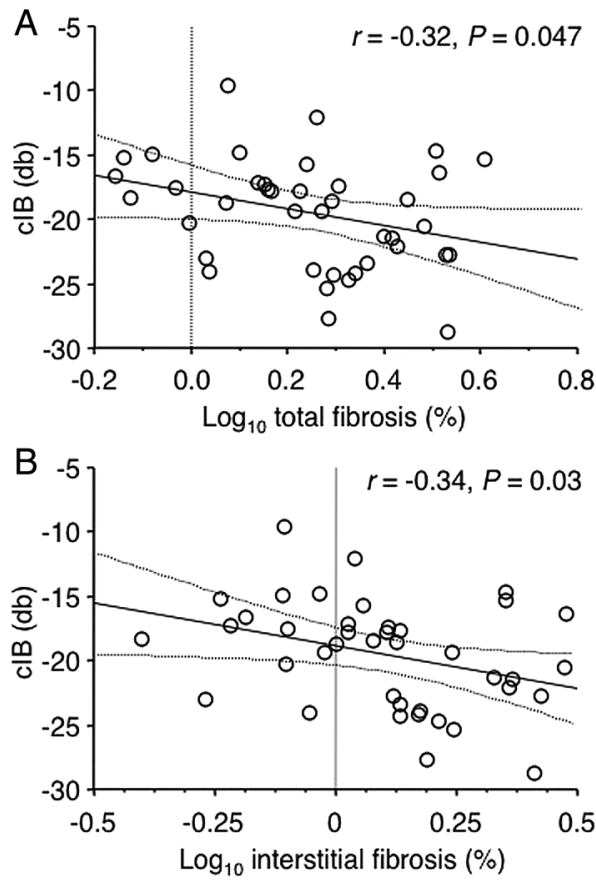

Figure 1 Correlation of calibrated integrated backscatter (cIB) with $\log _{10}$ total fibrosis (A) and $\log _{10}$ interstitial fibrosis (B) in patients undergoing coronary artery bypass graft surgery, $n=40$. The correlations of $\mathrm{ClB}$ with $\log _{10}$ total and interstitial fibrosis were also statistically significant using non-parametric Spearman correlations $(r=-0.32, p=0.04$ for total fibrosis; $r=-0.39, p=0.01$ for interstitial fibrosis).
I (PIP) or amino-terminal propeptides of procollagens type 1 and III (PINP and PIIINP) (table 1).

\section{Myocardial histology}

The median area of the myocardial sections was 4.0 (25th and 75th centiles were 2.9 and 6.8 , respectively) $\mathrm{mm}^{2}$. Total fibrosis, as assessed by picrosirius red staining, varied approximately 6 -fold, from $0.7 \%$ to $4 \%$, interstitial fibrosis varied 7.5 -fold from $0.4 \%$ to $3 \%$, and the perivascular fibrosis ratio varied 15 -fold from 0.3 to 4.6. cIB was weakly, but negatively correlated with $\log$ total and $\log$ interstitial fibrosis (figure 1), but was not correlated with perivascular fibrosis or any other histological parameter, including immunostaining for collagens I and III, advanced glycation end product (AGE) $\mathrm{N}^{\varepsilon}$-(carboxymethyl)lysine (CML) and the receptor for AGEs (RAGE) (table 3). Moreover, none of the other echocardiographic parameters (table 2) or PIP, PINP or PIIINP were significantly correlated with log total or log interstitial fibrosis (data not shown).

\section{Angiogenesis-related biomarkers}

Of the plasma angiogenesis-related biomarkers evaluated (table 4), $\log$ soluble vascular endothelial growth factor receptor-1 (sVEGFR-1) and log soluble RAGE (sRAGE) were associated with increased cIB (figure 2), but not with capillary length density (data not shown).

None of the plasma angiogenesis-related biomarkers evaluated (table 4) were correlated with total or interstitial myocardial fibrosis except for sRAGE, which was negatively correlated with $\log$ total fibrosis and $\log$ interstitial fibrosis using non-parametric Spearman correlations $(\mathrm{r}=-0.36, \mathrm{p}=0.04$ for total fibrosis; $\mathrm{r}=-0.50, \mathrm{p}=0.005$ for interstitial fibrosis). 
Table 3 Left ventricular histological parameters and their correlation with calibrated integrated backscatter values in 29 men and 11 women with coronary artery disease

\begin{tabular}{|c|c|c|c|}
\hline Variable & $\begin{array}{l}\text { Mean } \pm \text { SD or median } \\
\text { (IQR) }(n=40)\end{array}$ & $r$ & p Value \\
\hline Total fibrosis (\%) & $1.8(1.2-2.6)^{\star}$ & $-0.32 \dagger$ & $0.047 \dagger$ \\
\hline Interstitial fibrosis (\%) & $1.3(0.9-1.7)^{\star}$ & $-0.34 \dagger$ & $0.03 \dagger$ \\
\hline Perivascular fibrosis ratio & $1.7(1.1-2.3)^{\star}$ & $-0.12 \dagger$ & $0.49 \dagger$ \\
\hline Arterioles $/ \mathrm{mm}^{2}$ myocardium area & $0.8 \pm 0.4$ & -0.02 & 0.90 \\
\hline Mean arteriolar diameter $(\mu \mathrm{m})$ & $40 \pm 12$ & -0.17 & 0.31 \\
\hline Arteriolar wall area/circumference ratio $\left(\mu \mathrm{m}^{2} / \mu \mathrm{m}\right)$ & $5.5 \pm 1.7$ & -0.07 & 0.66 \\
\hline Cardiomyocyte width $(\mu \mathrm{m})$ & $22 \pm 3$ & -0.26 & 0.11 \\
\hline Capillary length density $\left(\mathrm{mm} / \mathrm{mm}^{3}\right)$ & $1106 \pm 419$ & 0.11 & 0.51 \\
\hline Diffusion radius $(\mu \mathrm{m})$ & $18 \pm 4$ & -0.12 & 0.47 \\
\hline Collagen I total fibrosis (\%) & $5.0 \pm 3.9$ & 0.06 & 0.74 \\
\hline Collagen I interstitial fibrosis (\%) & $2.4 \pm 2.8$ & 0.08 & 0.61 \\
\hline Collagen III total fibrosis (\%) & $10.0 \pm 4.6$ & -0.03 & 0.86 \\
\hline Collagen III interstitial fibrosis (\%) & $7.4 \pm 4.0$ & -0.02 & 0.92 \\
\hline Total collagen $1 / I I I$ ratio & $0.57 \pm 0.46$ & 0.12 & 0.48 \\
\hline Interstitial collagen I/III ratio & $0.35 \pm 0.27$ & 0.11 & 0.51 \\
\hline CML immunostaining arteriole media & $1.6 \pm 0.8$ & 0.15 & 0.36 \\
\hline CML immunostaining arteriole intima & $1.8 \pm 0.7$ & 0.07 & 0.65 \\
\hline RAGE immunostaining arteriole media & $0.6 \pm 0.7$ & -0.14 & 0.41 \\
\hline RAGE immunostaining arteriole intima & $1.6 \pm 0.8$ & -0.11 & 0.49 \\
\hline RAGE immunostaining capillaries & $1.3 \pm 1.0$ & 0.07 & 0.68 \\
\hline
\end{tabular}

\section{DISCUSSION}

The present study is the first to report the association between cIB and myocardial fibrosis in patients without extensive fibrosis. In contrast to previous studies of patients with dilated or hypertrophic cardiomyopathy and mean myocardial fibrosis of $15-34 \%,{ }^{1-5}$ we found that cIB was weakly but inversely associated with myocardial fibrosis of $0.7-4 \%$, as assessed by picrosirius red staining. However, cIB was not significantly associated with other histological parameters, including

Table 4 Plasma concentrations of angiogenesis-related biomarkers and their correlation with calibrated integrated backscatter values in 29 men and 11 women with coronary artery disease

\begin{tabular}{|c|c|c|c|}
\hline Variable & $\begin{array}{l}\text { Mean } \pm \text { SD or median (IQR) } \\
(n=33-40)\end{array}$ & $\mathbf{r}$ & p Value \\
\hline VEGF-A (pg/mL) & $22(13-33)^{\star}$ & $-0.17 \dagger$ & $0.34 \dagger$ \\
\hline sVEGFR-1 (pg/mL) & $90(64-131)^{\star}$ & $0.44 \dagger$ & $0.01 \dagger$ \\
\hline sVEGFR-2 (ng/mL) & $6.5 \pm 1.1$ & 0.07 & 0.70 \\
\hline Angiopoietin-1 (ng/mL) & $3.5(2.0-4.5)^{\star}$ & $-0.30 \dagger$ & $0.09 \dagger$ \\
\hline Angiopoietin-2 (ng/mL) & $1.4(1.2-1.9)^{*}$ & $0.29 \dagger$ & $0.10 \dagger$ \\
\hline Tie-1 (ng/mL) & $36 \pm 9$ & 0.04 & 0.82 \\
\hline Tie-2 (ng/mL) & $15 \pm 4$ & 0.14 & 0.44 \\
\hline Fibroblast growth factor basic ( $\mathrm{pg} / \mathrm{mL})$ & $6.8 \pm 2.9$ & -0.03 & 0.85 \\
\hline Endostatin $(\mathrm{ng} / \mathrm{mL})$ & $99 \pm 44$ & 0.16 & 0.38 \\
\hline Placental growth factor (pg/mL) & $11 \pm 4$ & 0.32 & 0.07 \\
\hline Hepatocyte growth factor (pg/mL) & $850(622-969)^{\star}$ & $0.26 \dagger$ & $0.14 \dagger$ \\
\hline $\mathrm{N}^{\varepsilon}$-(carboxymethyl)lysine $(\mu \mathrm{mol} / \mathrm{L})$ & $1.8 \pm 0.6$ & 0.12 & 0.46 \\
\hline LMWF (AU/mL) & $2.3(1.5-3.3)^{*}$ & $0.08 \dagger$ & $0.61 \dagger$ \\
\hline sRAGE & $527(456-937)^{\star}$ & $0.53 \dagger$ & $0.002 \dagger$ \\
\hline
\end{tabular}

${ }^{*}$ Median value and IQR given non-parametric distribution.

†Values derived from log-transformed data; $n=40$ for $\mathrm{N}^{\varepsilon}$-(carboxymethyl)lysine and LMWF levels, and $n=33$ for all other angiogenesis-related biomarkers.

LMWF, low-molecular-weight fluorophore; sRAGE, soluble receptor for advanced glycation end products; VEGF, vascular endothelial growth factor; sVEGFR, soluble VEGF receptor. 

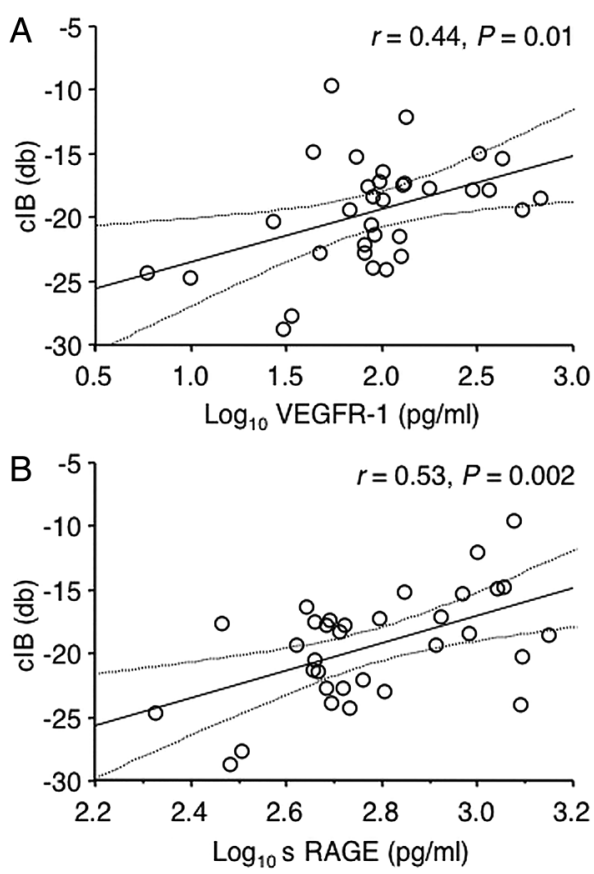

Figure 2 Correlations of calibrated integrated backscatter (clB) with $\log _{10}$ plasma soluble vascular endothelial growth factor receptor-1 (sVEGFR-1) (A), and $\log _{10}$ plasma soluble receptor for advanced glycation end products (sRAGE) (B) in patients undergoing coronary artery bypass graft surgery, $n=33$. The correlations of clB with $\log _{10}$ SVEGFR-1 and $\log _{10}$ sRAGE were also statistically significant using non-parametric Spearman correlations $(r=0.41, p=0.02$ for sVEGFR-1; $r=0.40$, $\mathrm{p}=0.02$ for $\mathrm{sRAGE}$ ).

immunostaining for collagens I and III, CML and RAGE. In addition, cIB was weakly associated with plasma levels of NT-proBNP, creatinine and eGFR, and more strongly associated with plasma sVEGFR-1 and sRAGE levels. We previously demonstrated less myocardial fibrosis in women than in men undergoing CABG surgery, ${ }^{18}$ and increased myocardial interstitial fibrosis in patients with aortic stenosis than in patients with CABG surgery without aortic stenosis. ${ }^{21}$ The present findings suggest that higher cIB values cannot be used as a surrogate measure of greater myocardial fibrosis in patients without dilated or hypertrophic cardiomyopathy and extensive fibrosis, and that potential surrogate markers of myocardial fibrosis need to be validated in different patient populations.

Although cIB is a measure of reflectance of the ultrasound signal by the myocardium, there is uncertainty about the determinants of cIB. The cIB values we measured were similar to those reported by Jellis $e t a l^{17}$ in a group of patients with early diabetic cardiomyopathy; their finding of only a borderline correlation between $\mathrm{T} 1$ values and $\operatorname{cIB}(p=0.053)$ is in agreement with our finding that higher cIB values were not a marker of greater fibrosis in patients without extensive fibrosis. Taken together with previous reports, ${ }^{1-5}$ our data suggest that the contribution of fibrosis to cIB depends on the extent of fibrosis and other factors play a greater role in determining cIB when fibrosis is less extensive. This may result in a threshold level of fibrosis below which cIB is not positively related to fibrosis, but above which it may be a useful measure. It is also possible that in previous studies, the observed relationship between cIB and fibrosis was, in fact, mediated by another property, and that this relationship was lost with less-extensive fibrosis. Our finding that cIB was associated with plasma NT-proBNP, creatinine and eGFR suggests that cIB is influenced by fluid status, and this is in agreement with previous reports that $\mathrm{CIB}$ is correlated with serum creatinine in patients with chronic renal failure ${ }^{12}$ and is reduced by increased hours/week of dialysis over 12 months. $^{13}$

The mechanism of the associations between cIB and plasma levels of sVEGFR-1 and sRAGE is not known. Increased plasma levels of sVEGFR-1 are associated with disease severity and adverse outcomes in chronic heart failure, ${ }^{22}$ and plasma sRAGE levels are associated with coronary events and cardiovascular mortality in diabetes. $^{23}{ }^{24}$ Thus, the basis of the association of plasma sVEGFR-1 and sRAGE levels with cIB may be similar to the association of acute myocardial ischaemia and acute cardiac allograft rejection with cIB. ${ }^{9}{ }^{10}$ sVEGFR-1 functions as a VEGF-A antagonist by preventing VEGF-A binding to VEGFR-2, the main VEGFR-mediating angiogenesis, and may thereby inhibit angiogenesis. It is also possible that the association of plasma levels of sVEGFR-1 and SRAGE with CIB has a similar basis to the reported association of plasma IL-13 levels with cIB in patients with idiopathic dilated cardiomyopathy. ${ }^{11}$ IL-13 causes antiinflammatory activities, ${ }^{25}$ and angiogenic and antiangiogenic activities have been reported for this cytokine. ${ }^{26} 27$ However, the failure of either sVEGFR-1 or sRAGE to correlate with myocardial capillary length density suggests that these markers represent mechanisms of myocardial reflectivity that are unrelated to angiogenesis.

There is uncertainty about the relevance of circulating collagen-derived peptides to cardiac fibrosis because these result from collagen turnover in many different tissues other than the heart. ${ }^{28}$ It is, therefore, not surprising that we found no correlation between PIP, PINP and PIIINP levels and either cIB or myocardial total or interstitial fibrosis. Jellis et $a l^{17}$ reported that PIIINP, but not PINP or PIP, was correlated with cIB; however, none were correlated with the postcontrast $\mathrm{T} 1$ value.

\section{Study limitations}

This was necessarily a cross-sectional study that provided limited information about the mechanisms of myocardial reflectivity because of the impossibility of obtaining serial myocardial biopsies, and our study was limited to 40 participants given the difficulty of obtaining myocardial biopsies in patients who were well characterised clinically, biochemically, haemodynamically and with cardiac imaging. This number of patients is, however, greater than the number studied in previous studies, ${ }^{1-35}$ except for the study of dilated cardiomyopathy by Mizuno et $a l^{4}$ Moreover, we studied biopsies from the same subepicardial 
region of the left ventricular myocardium in all patients and we do not know if the histology of these biopsies was representative of the regions used to estimate cIB. Although the patients had extensive coronary artery disease, we biopsied a region of myocardium that was free of ischaemia, without wall motion abnormalities; the histology of the biopsies showed no evidence of ischaemia or replacement fibrosis. We cannot exclude the possibility that myocardial interstitial and perivascular fibrosis were inhomogeneous, but our data clearly provide no support for a positive relationship between $\mathrm{cIB}$ and myocardial fibrosis in this patient population. Future studies are necessary to directly compare myocardial histology with alternative non-invasive methods of evaluation myocardial fibrosis, such as T1 mapping by cardiac MRI, ${ }^{17}$ and to investigate the relationships between cIB and plasma levels of NT-proBNP, creatinine, eGFR, sVEGFR-1 and sRAGE.

\section{CONCLUSION}

This study demonstrates that the positive association between cIB and myocardial fibrosis in patients with dilated or hypertrophic cardiomyopathy and extensive fibrosis cannot be extrapolated to patients with coronary artery disease without extensive fibrosis; by contrast, we found a weak inverse association between cIB and myocardial fibrosis in this patient population. cIB also showed weak associations with plasma levels of NT-proBNP, creatinine and eGFR, and stronger associations with plasma sVEGFR-1 and sRAGE levels. The role of cIB as a non-invasive index of fibrosis in clinical studies of patients with early myocardial disease without extensive fibrosis is, therefore, questionable.

\section{Author affiliations}

${ }^{1}$ Department of Cardiology, St. Vincent's Hospital Melbourne, Fitzroy, Australia

${ }^{2}$ Department of Medicine, University of Melbourne, St. Vincent's Hospital Melbourne, Fitzroy, Australia

${ }^{3}$ St. Vincent's Institute of Medical Research, Fitzroy, Australia

${ }^{4}$ Department of Cardiothoracic Surgery, St. Vincent's Hospital Melbourne, Fitzroy, Australia

${ }^{5}$ Department of Surgery, University of Melbourne, St. Vincent's Hospital Melbourne, Fitzroy, Australia

${ }^{6}$ Department of Internal Medicine, University of Maastricht, Maastricht, The Netherlands

${ }^{7}$ Department of Anatomy and Developmental Biology, Monash University, Clayton, Australia

Acknowledgements The authors thank Don Mooney for assistance with the performance of echocardiography; Laura Stamp, Robyn Kelly, Kim Hewitt and Francoise Campbell for assistance with histology; and Andrzej Januszewski for assistance with plasma assays.

Funding This study was funded by grants from the National Health and Medical Research Council of Australia (grant IDs 395508 and 566867) and from the National Heart Foundation of Australia (grant ID G08M 3833). St. Vincent's Institute of Medical Research is supported in part by the Victorian Government's Operational Infrastructure Support Program.

Competing interests None declared.

Ethics approval St. Vincent's Hospital Melbourne Human Research Ethics Committee.
Provenance and peer review Not commissioned; externally peer reviewed.

Data sharing statement De-identified individual patient data will be made available to any researcher who requests such data.

Open Access This is an Open Access article distributed in accordance with the Creative Commons Attribution Non Commercial (CC BY-NC 4.0) license, which permits others to distribute, remix, adapt, build upon this work noncommercially, and license their derivative works on different terms, provided the original work is properly cited and the use is non-commercial. See: http:// creativecommons.org/licenses/by-nc/4.0/

\section{REFERENCES}

1. Picano $\mathrm{E}$, Pelosi $\mathrm{G}$, Marzilli $\mathrm{M}$, et al. In vivo quantitative ultrasonic evaluation of myocardial fibrosis in humans. Circulation 1990;81:58-64.

2. Naito J, Masuyama T, Mano T, et al. Ultrasonic myocardial tissue characterization in patients with dilated cardiomyopathy: value in noninvasive assessment of myocardial fibrosis. Am Heart $J$ 1996;131:115-21.

3. Di Bello V, Giorgi D, Viacava P, et al. Severe aortic stenosis and myocardial function: diagnostic and prognostic usefulness of ultrasonic integrated backscatter analysis. Circulation 2004;110:849-55.

4. Mizuno R, Fujimoto S, Saito $\mathrm{Y}$, et al. Non-invasive quantitation of myocardial fibrosis using combined tissue harmonic imaging and integrated backscatter analysis in dilated cardiomyopathy. Cardiology 2007;108:11-17.

5. Mizuno R, Fujimoto S, Yamaji K, et al. Myocardial ultrasonic tissue characterization for estimating histological abnormalities in hypertrophic cardiomyopathy: comparison with endomyocardial biopsy findings. Cardiology 2001;96:16-23.

6. Jellis CL, Stanton T, Leano R, et al. Usefulness of at rest and exercise hemodynamics to detect subclinical myocardial disease in type 2 diabetes mellitus. Am J Cardiol 2011;107:615-21.

7. Kosmala W, Przewlocka-Kosmala M, Wojnalowicz A, et al. Integrated backscatter as a fibrosis marker in the metabolic syndrome: association with biochemical evidence of fibrosis and left ventricular dysfunction. Eur Heart $J$ Cardiovasc Imaging 2012;13:459-67.

8. Campbell DJ, Somaratne JB, Jenkins AJ, et al. Impact of type 2 diabetes and the metabolic syndrome on myocardial structure and microvasculature of men with coronary artery disease. Cardiovasc Diabetol 2011;10:80.

9. Picano E, Faletra F, Marini C, et al. Increased echodensity of transiently asynergic myocardium in humans: a novel echocardiographic sign of myocardial ischemia. J Am Coll Cardiol 1993;21:199-207.

10. Angermann CE, Nassau K, Stempfle HU, et al. Recognition of acute cardiac allograft rejection from serial integrated backscatter analyses in human orthotopic heart transplant recipients. Comparison with conventional echocardiography. Circulation 1997;95:140-50.

11. Ohtsuka T, Inoue K, Hara Y, et al. Serum markers of angiogenesis and myocardial ultrasonic tissue characterization in patients with dilated cardiomyopathy. Eur J Heart Fail 2005;7:689-95.

12. Salvetti M, Muiesan ML, Paini A, et al. Myocardial ultrasound tissue characterization in patients with chronic renal failure. J Am Soc Nephrol 2007;18:1953-8.

13. Jin X, Rong S, Mei C, et al. Effects of thrice-weekly in-center nocturnal vs. conventional hemodialysis on integrated backscatter of myocardial tissue. Hemodial Int 2011;15:200-10.

14. Minczykowski A, Zaremba-Drobnik D, Pietrzak I, et al. Effect of preload reduction on sonographic myocardial integrated backscatter. J Clin Ultrasound 2008;36:157-65.

15. Wong CY, O'Moore-Sullivan T, Leano R, et al. Alterations of left ventricular myocardial characteristics associated with obesity. Circulation 2004;110:3081-7.

16. Wong CY, Byrne NM, O'Moore-Sullivan T, et al. Effect of weight loss due to lifestyle intervention on subclinical cardiovascular dysfunction in obesity (body mass index $>30 \mathrm{~kg} / \mathrm{m} 2$ ). Am J Cardiol 2006;98:1593-8.

17. Jellis $C$, Wright $J$, Kennedy $D$, et al. Association of imaging markers of myocardial fibrosis with metabolic and functional disturbances in early diabetic cardiomyopathy. Circ Cardiovasc Imaging 2011;4:693-702.

18. Campbell DJ, Somaratne JB, Jenkins AJ, et al. Differences in myocardial structure and coronary microvasculature between men and women with coronary artery disease. Hypertension 2011;57:186-92. 
19. Lang RM, Bierig M, Devereux RB, et al. Recommendations for chamber quantification: a report from the American Society of Echocardiography's Guidelines and Standards Committee and the Chamber Quantification Writing Group, developed in conjunction with the European Association of Echocardiography, a branch of the European Society of Cardiology. $J$ Am Soc Echocardiogr 2005;18:1440-63.

20. Quinones MA, Otto CM, Stoddard M, et al. Recommendations for quantification of Doppler echocardiography: a report from the Doppler Quantification Task Force of the Nomenclature and Standards Committee of the American Society of Echocardiography. J Am Soc Echocardiogr 2002;15:167-84.

21. Campbell DJ, Somaratne JB, Jenkins AJ, et al. Reduced microvascular density in non-ischemic myocardium of patients with recent non-ST-segment-elevation myocardial infarction. Int J Cardiol 2013;167:1027-37.

22. Ky B, French B, Ruparel K, et al. The vascular marker soluble fms-like tyrosine kinase 1 is associated with disease severity and adverse outcomes in chronic heart failure. J Am Coll Cardiol 2011;58:386-94

23. Nin JW, Jorsal A, Ferreira I, et al. Higher plasma soluble receptor for advanced glycation end products (SRAGE) levels are associated with incident cardiovascular disease and all-cause mortality in type 1 diabetes: a 12-year follow-up study. Diabetes 2010;59:2027-32.
24. Colhoun HM, Betteridge DJ, Durrington $P$, et al. Total soluble and endogenous secretory receptor for advanced glycation end products as predictive biomarkers of coronary heart disease risk in patients with type 2 diabetes: an analysis from the CARDS trial. Diabetes 2011;60:2379-85

25. Minty A, Chalon P, Derocq JM, et al. Interleukin-13 is a new human lymphokine regulating inflammatory and immune responses. Nature 1993;362:248-50.

26. Fukushi J, Ono M, Morikawa W, et al. The activity of soluble VCAM-1 in angiogenesis stimulated by IL-4 and IL-13. J Immunol 2000;165:2818-23.

27. Nishimura $\mathrm{Y}$, Nitto $\mathrm{T}$, Inoue $\mathrm{T}$, et al. IL-13 attenuates vascular tube formation via JAK2-STAT6 pathway. Circ J 2008;72:469-75.

28. Lopez B, Gonzalez A, Diez J. Circulating biomarkers of collagen metabolism in cardiac diseases. Circulation 2010;121:1645-54

29. Levey AS, Bosch JP, Lewis JB, et al. A more accurate method to estimate glomerular filtration rate from serum creatinine: a new prediction equation. Modification of Diet in Renal Disease Study Group. Ann Intern Med 1999;130:461-70.

30. Wallace TM, Levy JC, Matthews DR. Use and abuse of HOMA modeling. Diabetes Care 2004;27:1487-95. 